\title{
Dense Cranial Electroacupuncture Stimulation for Major Depressive Disorder-A Single-Blind, Randomized, Controlled Study
}

\author{
Zhang-Jin Zhang ${ }^{1 *}$, Roger $\mathrm{Ng}^{1}$, Sui Cheung Man ${ }^{1}$, Tsui Yin Jade $\mathrm{Li}^{1}$, Wendy Wong ${ }^{1}$, Qing-Rong Tan ${ }^{3}$, Hei \\ Kiu Wong ${ }^{1}$, Ka-Fai Chung ${ }^{4}$, Man-Tak Wong ${ }^{2}$, Wai-Kiu Alfert Tsang ${ }^{2}$, Ka-chee Yip ${ }^{2}$, Eric Ziea ${ }^{5}$, Vivian Taam \\ Wong $^{5}$
}

1 School of Chinese Medicine, LKS Faculty of Medicine, The University of Hong Kong, Hong Kong, China, 2 Department of Psychiatry, Kowloon Hospital, Hong Kong, China, 3 Department of Psychiatry, Fourth Military Medical University, Xi'an, Shaanxi, China, 4 Department of Psychiatry, LKS Faculty of Medicine, The University of Hong Kong, Hong Kong, China, $\mathbf{5}$ Chinese Medicine Section, Hospital Authority, Hong Kong, China

\begin{abstract}
Background: Previous studies suggest that electroacupuncture possesses therapeutic benefits for depressive disorders. The purpose of this study was to determine whether dense cranial electroacupuncture stimulation (DCEAS) could enhance the antidepressant efficacy in the early phase of selective serotonin reuptake inhibitor (SSRI) treatment of major depressive disorder (MDD).

Methods: In this single-blind, randomized, controlled study, patients with MDD were randomly assigned to 9-session DCEAS or noninvasive electroacupuncture (n-EA) control procedure in combination with fluoxetine (FLX) for 3 weeks. Clinical outcomes were measured using the 17-item Hamilton Depression Rating Scale (HAMD-17), Clinical Global Impressionseverity (CGI-S), and Self-rating Depression Scale (SDS) as well as the response and remission rates.

Results: Seventy-three patients were randomly assigned to $n$-EA $(n=35)$ and DCEAS $(n=38)$, of whom 34 in $n$-EA and 36 in DCEAS group were analyzed. DCEAS-treated patients displayed a significantly greater reduction from baseline in HAMD-17 scores at Day 3 through Day 21 and in SDS scores at Day 3 and Day 21 compared to patients receiving n-EA. DCEAS intervention also produced a higher rate of clinically significant response compared to n-EA procedure $(19.4 \%(7 / 36)$ vs. $8.8 \%(3 / 34))$. The incidence of adverse events was similar in the two groups.

Conclusions: DCEAS is a safe and effective intervention that augments the antidepressant efficacy. It can be considered as an additional therapy in the early phase of SSRI treatment of depressed patients.

\section{Trial Registration: Controlled-Trials.com ISRCTN88008690}

Citation: Zhang Z-J, Ng R, Man SC, Li TYJ, Wong W, et al. (2012) Dense Cranial Electroacupuncture Stimulation for Major Depressive Disorder-A Single-Blind, Randomized, Controlled Study. PLoS ONE 7(1): e29651. doi:10.1371/journal.pone.0029651

Editor: Kenji Hashimoto, Chiba University Center for Forensic Mental Health, Japan

Received September 7, 2011; Accepted November 30, 2011; Published January 6, 2012

Copyright: (c) 2012 Zhang et al. This is an open-access article distributed under the terms of the Creative Commons Attribution License, which permits unrestricted use, distribution, and reproduction in any medium, provided the original author and source are credited.

Funding: This study was supported by Health and Health Services Research Fund (HHSRF) from Food and Health Bureau of Hong Kong (ref. No.: 06070831). The funders had no role in study design, data collection and analysis, decision to publish, or preparation of the manuscript.

Competing Interests: The authors have declared that no competing interests exist.

*E-mail: zhangzj@hku.hk
\end{abstract}

\section{Introduction}

Although selective serotonin reuptake inhibitors (SSRIs) are the mainstay in the treatment of depressive disorders, the treatment outcomes are unsatisfactory [1]. There remains a large portion of depressed patients who cannot obtain a full remission and experience relapse and functional impairment [1,2]. Moreover, the delay in the onset of the action of SSRIs prolongs patients' suffering and exposes them to great risk of suicide [3]. These shortcomings have led to a high demand for seeking alternative strategies that can enhance the antidepressant efficacy of SSRIs particularly in the early phase of the treatment [4].

Numerous studies and recent meta-analyses have shown that acupuncture is efficacious for various types of depressive disorders
[5-7]. Although most acupuncture protocols used were developed from the doctrine of traditional Chinese medicine and empiricism rather than modern scientific rationale, experimental and clinical observations have found that electroacupuncture has robust immediate and short-term effects in alleviating pain, autonomic dysfunction, sleep, and mood symptoms [8-10]. This rapid effect is thought to be associated with the fast and direct modulation of multiple central neurochemical systems, especially the brainstem adrenalinergic (NA), serotonergic (5-HT) neuronal and hypothalamic neuroendocrine systems [10], which play the principal role in the pathophysiology of major depression [11]. These are the reasons to hypothesize that electroacupuncture can serve to enhance the antidepressant action in the early phase of SSRI treatment. 
Dense cranial electroacupuncture stimulation (DCEAS) is a novel stimulation mode in which electrical stimulation is delivered on dense acupoints located on the forehead mainly innervated by the trigeminal nerve, efficiently modulating multiple central transmitter systems via the trigeminal sensory-brainstem NA and 5-HT neuronal pathways [12]. Several pilot studies have shown that DCEAS and similar approaches are effective in improving refractory obsessive-compulsive disorder (OCD) [12], major depressive disorder (MDD) [13], post-stroke depression [14], and MDD-associated residual insomnia [15].

Fluoxetine (FLX) is one of the most prescribed SSRIs for major depression worldwide [16]. This single-blind, randomized, controlled trial was designed to determine whether DCEAS intervention could produce greater clinical improvement compared to noninvasive electroacupuncture (n-EA) control procedure in the early phase of FLX treatment of patients with MDD.

\section{Methods}

\section{Subjects}

This single-blind, randomized, sham-acupuncture controlled trial was conducted in Department of Psychiatry at Kowloon Hospital of Hong Kong between August 2009 and March 2011. The study protocol was approved by Institutional Review Board (IRB) of the University of Hong Kong/Hospital Authority Hong Kong West Cluster and registered in www.controlled-trials.com (ISRCTN88008690). The protocol for this trial and supporting CONSORT checklist are available as supporting information (see Checklist S1 and Protocol S1).

Psychiatrists referred outpatients to the study. The inclusion criteria were: (1) age 25-65 years; (2) DSM-IV diagnosis of MDD [17]; (3) 17-item Hamilton Rating Scale for Depression (HAMD17) score $\geq 18$ [18]; and (4) Clinical Global Impression-Severity (GGI-S) score $\geq 4$ [19]. Subjects were excluded if they had: (1) unstable medical conditions; (2) suicidal attempts or aggressive behavior; (3) a history of manic, hypomanic, or mixed episode; (4) a family history of bipolar or psychotic disorders; (5) a history of substance abuse within the previous 12 months; (6) investigational drug treatment in the previous 6 months; (7) current psychotropic treatment exceed one week; or (8) needle phobia. All participants gave voluntary, written and informed consent before entering the trial.

\section{Randomization and blinding}

Patients were randomly assigned to either n-EA or DCEAS treatment at a ratio of $1: 1$, using a random block scheme from an automatic computer program (SPSS version II). The assignment was done in a single-blind manner, in which the random codes were only known by the acupuncturists (W.W. and M. S.C.). The validity of the subject-blind design was ensured by sham acupuncture procedure performed on the forehead acupoints, which were outside the visual field of the subjects (see below). In order to minimize the expected effects, patients were not told about the potential response of control and DCEAS procedure during random assignment.

\section{Fluoxetine treatment}

Unmedicated patients in both groups received orally administered FLX for 3 weeks in an open manner. FLX dose was initiated at $10 \mathrm{mg} /$ day and escalated to an optimal dose within one week based on individual patients' response, with a maximum dose of $40 \mathrm{mg} /$ day. Attaining a balance of efficacy and side effects, this FLX dosing regimen has been widely used in previous studies of major depression in the Chinese population [20,21]. Those who were currently treated with FLX for no more than one week continued their FLX treatment with the same dose. Those who were currently treated with other psychotropic medications for no more than one week were required to be switched to the FLX regimen by gradually withdrawing the drugs within one week in order to wash out potential "carryover" effects. The information about the equivalent efficacy of FLX was offered to the patients. Concomitant use of other psychotropic drugs was not allowed. Medication compliance was determined by pill count at each study visit. Patients who required concomitant medications and those having less than $80 \%$ FLX compliance were advised to withdraw from the study.

\section{DCEAS and n-EA procedure}

The patients received 9 sessions of $\mathrm{n}$-EA or DCEAS intervention (3 sessions per week) during FLX treatment. Electrical stimulation was delivered on the following 6 matches of forehead acupoints that are innervated by the trigeminal nerve via inserted or non-inserted needles (Fig. 1): Baihui (Du-20) and Yintang (EXHN3), left Sishencong (EX-HNl) and Toulinqi (GB15), right Sishencong (EX-HN1) and Toulinqi (GB15), bilateral Shuaigu (GB8), bilateral Taiyang (EX-HN5), and bilateral Touwei (ST8). For DCEAS, disposable acupuncture needles $(0.30 \mathrm{~mm}$ in diameter and 25-40 $\mathrm{mm}$ in length) were inserted into acupoints for a depth of $10-30 \mathrm{~mm}$ in a direction oblique or parallel to the surface. To ensure allocation concealment, the inserted needles were affixed with adhesive tapes so that DCEAS procedure was identical to control acupuncture procedure. Electrical stimulation with continuous waves at $2 \mathrm{~Hz}$ and constant current and voltage $(9 \mathrm{~V})$ was delivered via an acupuncture stimulation instrument (Hwarto, SDZ-II) for 30 min (the pulse width could not be determined in this model instrument). The choice of this stimulation mode was based on the fact that low frequency could exert broader effects on central neurochemical systems compared to high frequency and has been widely introduced into the treatment of neuropsychiatric disorders $[10,22]$. The intensity of stimulation was adjusted to a level at which the patients felt most comfortable. For n-EA procedure, Streitberger's noninvasive acupuncture needles were used [23,24]. Its validity and credibility have been well demonstrated [23,24]. The needles with blunt tips were quickly put onto the same acupoints used in DCEAS without inserting into the skin. The needles were then affixed with plastic O-rings and adhesive tapes. Electrical stimulation was delivered with the same parameters as DCEAS. Patients felt the stimulation via blunt tips touched on the skin.

To ensure consistency in acupuncture procedure, the principal investigator (Z.J.Z.) provided a training workshop of acupuncture protocol. Acupuncture intervention was performed by registered acupuncturists (W.W. and S.C.M.) who had received 5-year undergraduate training in Chinese medicine and had practiced Chinese medicine over three years.

\section{Assessment}

Treatment outcomes were changes from baseline in the total score on HAMD-17 [18], CGI-S [19], and the Chinese-version Self-rating Depression Scale (SDS) [25] at baseline and at day 3, 7, 14 , and 21 . The secondary outcome measures included treatment response, defined as $\geq 50 \%$ reduction at endpoint from baseline on HAMD-17, and remission, defined as an endpoint HAMD-17 score of $\leq 7$. Safety and tolerability were assessed using the Treatment Emergent Symptom Scale (TESS) [19], in which adverse events were recorded at each visit, including their date and time of onset, duration, severity, relationship to intervention, and the action taken. 


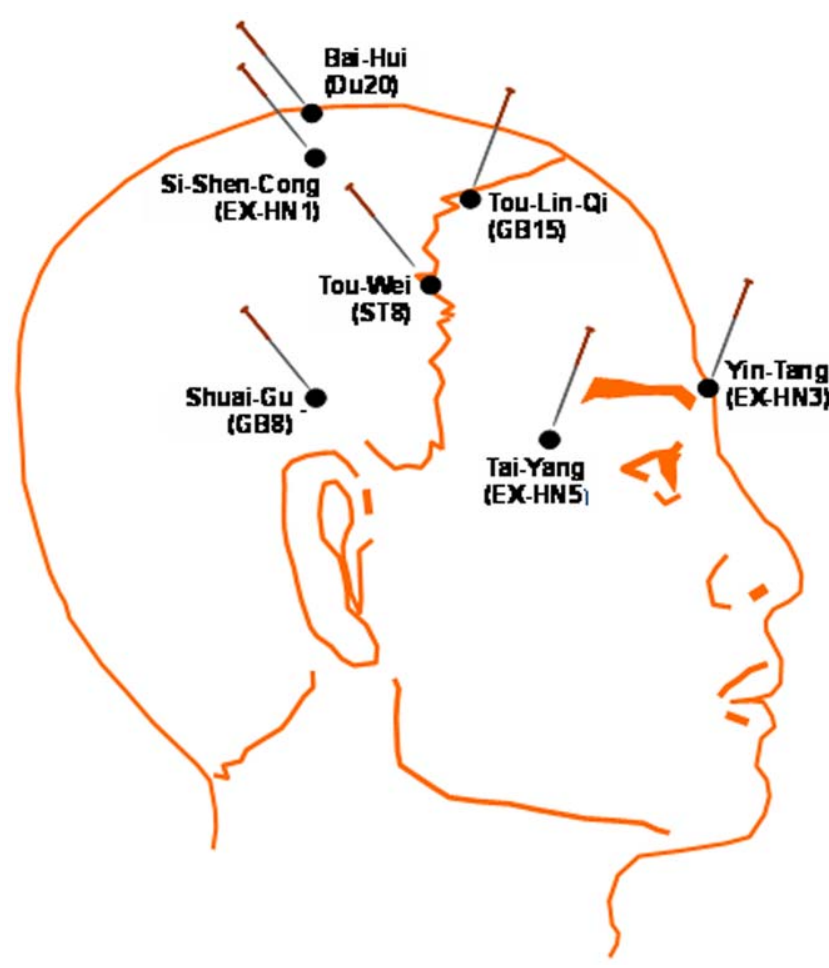

Figure 1. Acupoints used in dense cranial electroacupuncture stimulation (DCEAS).

doi:10.1371/journal.pone.0029651.g001

Both patients and raters were blind to the treatment allocation. A training workshop with video materials was conducted for raters who might be involved in clinical assessments. An interrater reliability coefficient ( $\kappa$ value) of $>0.80$ was achieved after the completion of training workshop. In this study, all assessments were completed by the same rater (J.L.).

The credibility of n-EA and DCEAS procedure was evaluated based on Fink et al. method [26] by asking the patients: "As we informed you that you had an equal chance of receiving sham or active acupuncture treatment, which do you think you had received?"

\section{Statistical analysis}

Based on our recent meta-analysis, a sample size of 70 patients ( $n=35$ per group) could provide approximately $80 \%$ power to detect an estimated difference in HAMD- 17 score of 3 points, with $\alpha$ set at 0.05 and an estimated standard deviation of 4.5 at the endpoint of 3-week treatment [5].

Efficacy analyses were performed on the intention-to-treat population, defined as participants who completed baseline and at least one evaluation after treatment. Since measure time points were not balanced, a linear mixed-effects model was preferably applied to compare treatment outcomes (HAMD-17, CGI-S and SDS) over time between the two groups. The model was established using time and group for categorical fixed factors and random intercepts with scaled identity covariance matrix. Subject's age, gender, duration of the illness, number of relapse, baseline HAMD-17, tolerability and credibility for acupuncture procedure were treated as covariates. Between-group differences at each measure time point were examined using Student $t$-test. The data was expressed as mean with $95 \%$ confidence interval $(95 \%$ CI). Student $t$ test was used to compare continuous baseline variables between the two groups. Categorical variables, including categorical baseline variables, response and remission rates, incidence of adverse events, treatment compliance, and credibility, were analyzed using Chi-square $\left(\chi^{2}\right)$ test or Fisher exact test if one or more expected frequencies were less than 5. Statistical significance was defined as a two-sided $P<0.05$. The analyses were performed with SPSS version 16 software (Chicago, IL, USA).

\section{Results}

\section{Disposition and characteristics of patients}

Of 188 outpatients referred by psychiatrists for screening, 73 eligible patients were randomly assigned to n-EA $(n=35)$ and DCEAS (n = 38) group; while $63(86.3 \%)$ of them completed the 3week assessment. One patient in n-EA group was excluded from analysis, because she was later found to have cocaine use in the past year. Two patients in DCEAS group were excluded from analysis due to a lack of post-baseline assessment. Seventy patients (34 in n-EA and 36 in DCEAS) were included in data analysis (Fig. 2).

Baseline characteristics of patients are summarized in Table 1. The proportion of females assigned to n-EA group was significantly higher than that in DCEAS group $(97.1 \%$ vs. $69.4 \%, P=0.006$, Chi-square test). Other baseline variables were similar in the two groups. Nearly $93 \%(65 / 70)$ patients had experienced relapses and $66 \%(46 / 70)$ patients had acupuncture treatment previously. There were only $18.6 \%$ (13/70) of patients receiving psychotropic medication when entering the study. The compliance with acupuncture and FLX treatment was nearly $95 \%$ in the two groups.

\section{Efficacy}

Changes from baseline in score on HAMD-17, CGI-S, and SDS over time are illustrated in Table 2 and Fig. 3. The analyses based on linear mixed-effects model revealed highly linear correlations between measure time points and changes from baseline in score on HAMD $\left(r^{2}=0.497\right.$ in n-EA group and $r^{2}=0.531$ in DCEAS group, $P<0.0001)$, CGI $\left(r^{2}=0.400\right.$ in n-EA group and $r^{2}=0.381$ in DCEAS group, $P<0.0001)$, and $\operatorname{SDS}\left(r^{2}=0.192\right.$ in n-EA group and $r^{2}=0.248$ in DCEAS group, $\left.P<0.0001\right)$. There were significant differences in the slope and/or intercept between nEA and DCEAS groups on HAMD $(F=5.938$, df $=1,336$, $P=0.015)$ and SDS $(F=5.885, \mathrm{df}=1,336, P=0.016)$, but not CGI $(F=232, \mathrm{df}=1,336, P=0.631)$. Between-group comparisons further revealed that DCEAS-treated patients had a significantly greater reduction in scores on HAMD-17 compared to patients receiving n-EA procedure at Day 3 through Day 21 ( $P \leq 0.025)$. The significantly greater reduction was also observed in SDS scores at day $3(P=0.037)$ and day $21(P=0.004)$.

The response rate in DCEAS group was not significantly different from that in n-EA group $(19.4 \%(7 / 36)$ vs. $8.8 \%(3 / 34)$, $P=0.308$, Fisher Exact test). The remission rate was also similar in the two groups $(2.7 \%(1 / 36)$ vs. $2.9 \%(1 / 34), P=0.998$, Fisher Exact test).

The average dose of FLX in DCEAS group was similar to that in n-EA group $(23.01 \pm 3.2 \mathrm{mg} /$ day (mean $\pm \mathrm{SD})$ vs. $23.4 \pm 2.4 \mathrm{mg} /$ day, $P=0.599, t$-test).

\section{Safety and tolerability}

Adverse events occurred in at least $5 \%$ of the patients in either group are listed in Table 3. No significant differences in the incidence of any adverse events were found between the two groups. There were $20.6 \%$ (7/34) of patients who felt uncomfortable in n-EA procedure, but not significantly different from $38.9 \%$ 


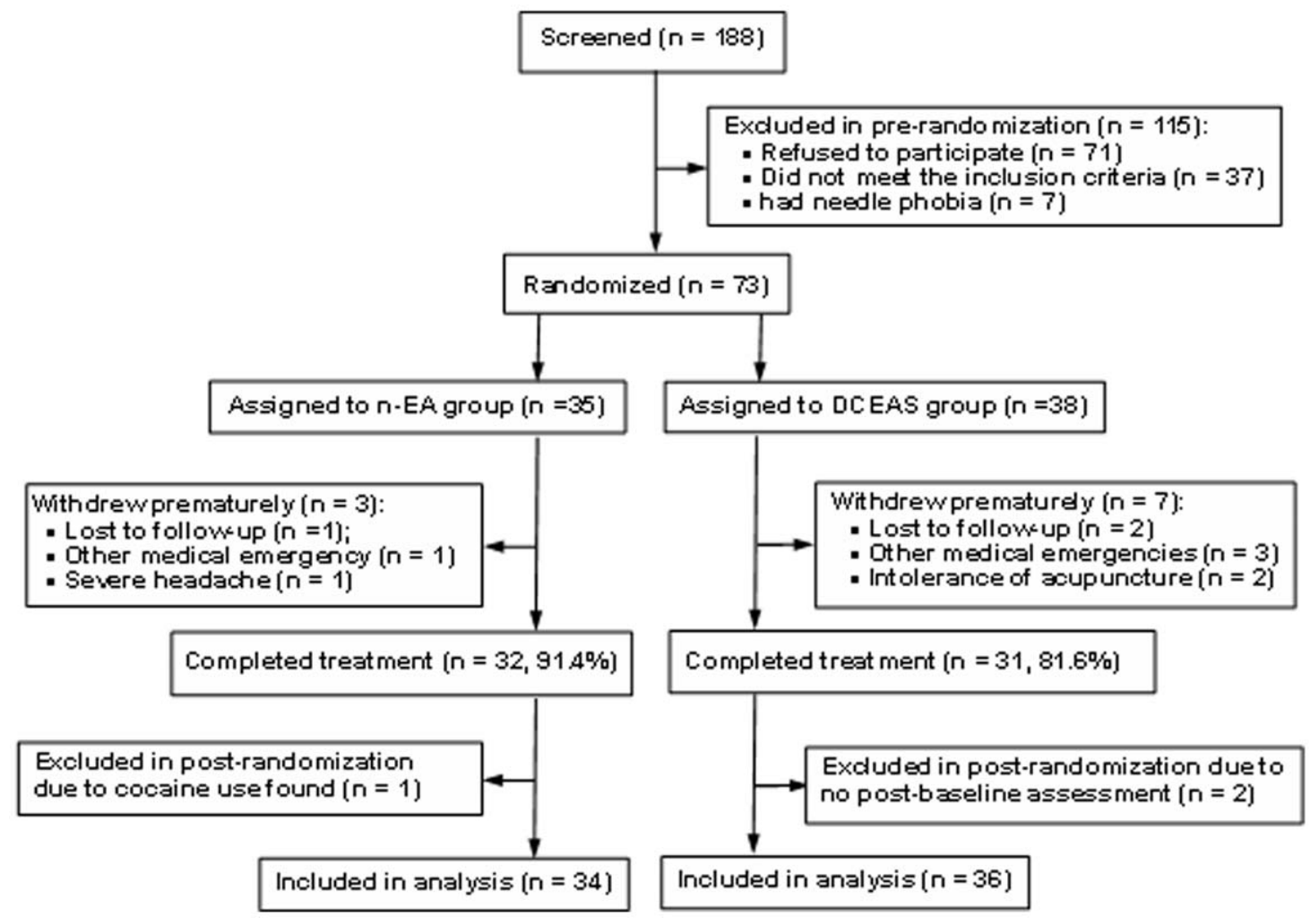

Figure 2. Flowchart of screening and patient recruitment. $n-E A$, noninvasive electroacupuncture; DCEAS, dense cranial electroacupuncture stimulation.

doi:10.1371/journal.pone.0029651.g002

Table 1. Baseline characteristics of patients.

\begin{tabular}{|c|c|c|c|}
\hline Variables & $\begin{array}{l}n-E A \\
(n=34)\end{array}$ & $\begin{array}{l}\text { DCEAS } \\
(n=36)\end{array}$ & $\begin{array}{l}P \text { values } \\
\left(t \text { or } \chi^{2} \text { test) }\right.\end{array}$ \\
\hline Female, n (\%) & $33(97.1)$ & $25(69.4)$ & 0.006 \\
\hline Age $(y r s)^{a}$ & $48.2 \pm 9.8$ & $46.3 \pm 9.9$ & 0.414 \\
\hline Duration of MDD (yrs) ${ }^{\mathrm{a}}$ & $7.3 \pm 7.1$ & $7.9 \pm 8.0$ & 0.744 \\
\hline No. of previous depressive episodes ${ }^{a}$ & $3.6 \pm 4.4$ & $4.9 \pm 6.1$ & 0.332 \\
\hline No. (\%) of patients with first-onset MDD & $3(8.8)$ & $2(5.5)$ & 0.669 \\
\hline No. (\%) of patients with previous psychiatric admission & $8(23.5)$ & $7(19.4)$ & 0.901 \\
\hline No. (\%) of patients with family members having mental illnesses. & $9(26.5)$ & $13(36.1)$ & 0.800 \\
\hline No. (\%) of patients with previous acupuncture treatment ${ }^{\mathrm{b}}$ & $22(64.7)$ & $24(66.7)$ & 0.937 \\
\hline No. (\%) of patients receiving psychotropic medications at study entry ${ }^{c}$ & $6(17.6)$ & $7(19.4)$ & 0.909 \\
\hline SSRIs & 3 & 3 & \\
\hline SNRIs & 1 & 1 & \\
\hline Mood stabilizers & $1^{d}$ & 1 & \\
\hline Benzodiazepines & 2 & 2 & \\
\hline Baseline HAMD-17 score ${ }^{a}$ & $23.1 \pm 3.6$ & $23.9 \pm 3.8$ & 0.321 \\
\hline Baseline $\mathrm{CGI}^{\mathrm{a}} \mathrm{S}^{\mathrm{a}}$ & $4.3 \pm 0.5$ & $4.4 \pm 0.5$ & 0.760 \\
\hline Baseline SDS score ${ }^{\mathrm{a}}$ & $40.6 \pm 14.5$ & $41.9 \pm 14.0$ & 0.704 \\
\hline
\end{tabular}

${ }^{\mathrm{a}}$ Continuous data are expressed as mean $\pm \mathrm{SD}$.

${ }^{\mathrm{b}}$ Auricular acupuncture was included.

'The use of medications did not exceed one week.

dOne patient received a combination of SNRIs and mood stabilizers.

n-EA, noninvasive electroacupuncture; DCEAS, dense cranial electroacupuncture stimulation; MDD, major depressive disorder; SSRIs, selective serotonin re-uptake

inhibitors; SNRIs, Serotonin-norepinephrine reuptake inhibitors; HAMD, 17-item Hamilton Rating Scale for Depression; CGI-S, Clinical Global Impression-Severity; SDS, Self-rating Depression Scale.

doi:10.1371/journal.pone.0029651.t001 
Table 2. Changes in score on depression scales from baseline in MDD patients.

\begin{tabular}{|c|c|c|c|c|c|}
\hline Variables & $\begin{array}{l}\text { n-EA }(n=34) \\
(95 \% \mathrm{Cl})\end{array}$ & $\begin{array}{l}\text { DCEAS }(n=36) \\
(95 \% \mathrm{Cl})\end{array}$ & $\begin{array}{l}\text { Between-group } \\
\text { difference }(95 \% \mathrm{Cl})\end{array}$ & $\begin{array}{l}\text { Overall } \\
P \text { value }^{\mathrm{a}}\end{array}$ & $\begin{array}{l}\text { Between-group } \\
P \text { value }^{\mathrm{a}}\end{array}$ \\
\hline HAMD-17 & & & & 0.015 & \\
\hline Day 3 & $-3.71(-4.34--3.06)$ & $-5.97(-6.71--5.23)$ & $2.27(1.29-3.25)$ & & 0.000 \\
\hline Day 7 & $-5.82(-6.46--5.18)$ & $-6.97(-7.71--6.23)$ & $1.15(0.17-2.13)$ & & 0.025 \\
\hline Day 14 & $-6.41(-7.05--5.77)$ & $-8.44(-9.18--7.70)$ & $2.03(1.05-3.01)$ & & 0.000 \\
\hline Day 21 & $-6.27(-6.90--5.62)$ & $-8.66(-9.39--7.91)$ & $2.39(1.41-3.37)$ & & 0.000 \\
\hline CGI-S & & & & 0.631 & \\
\hline Day 3 & $-0.32(-0.42--0.22)$ & $-0.44(-0.54--0.34)$ & $0.12(-0.03-0.27)$ & & 0.116 \\
\hline Day 7 & $-0.65(-0.75--0.55)$ & $-0.53(-0.63--0.43)$ & $0.12(-0.03-0.27)$ & & 0.116 \\
\hline Day 14 & $-0.71(-0.81--0.61)$ & $-0.71(-0.81--0.61)$ & $0.00(-0.15-0.15)$ & & 1.000 \\
\hline Day 21 & $-0.74(-0.84--0.64)$ & $-0.74(-0.84--0.64)$ & $0.00(-0.15-0.15)$ & & 1.000 \\
\hline SDS & & & & 0.016 & \\
\hline Day 3 & $-6.44(-8.48--4.40)$ & $-9.76(-12.03--7.49)$ & $3.32(0.26-6.38)$ & & 0.037 \\
\hline Day 7 & $-8.82(-10.86--6.78)$ & $-9.12(-11.39--6.85)$ & $0.30(-2.76-3.36)$ & & 0.851 \\
\hline Day 14 & $-11.74(-13.78--9.70)$ & $-12.38(-14.65--10.11)$ & $0.64(-2.42-3.70)$ & & 0.679 \\
\hline Day 21 & $-8.38(-10.42--6.34)$ & $-13.06(-15.33--10.79)$ & $4.68(1.62-7.74)$ & & 0.004 \\
\hline
\end{tabular}

${ }^{a}$ Overall and between-group $P$ values were obtained from linear mixed-effects model analysis and student $t$-test, respectively.

MDD, major depressive disorder; n-EA, noninvasive electroacupuncture; DCEAS, dense cranial electroacupuncture stimulation; $95 \% \mathrm{Cl}$, $95 \%$ confidence interval; $\mathrm{HAMD-}$ 17, 17-item Hamilton Rating Scale for Depression; CGI-S, Clinical Global Impression-Severity; SDS, Self-rating Depression Scale.

doi:10.1371/journal.pone.0029651.t002

$(14 / 36)$ in DCEAS-treated patients $\left(\chi^{2}=1.985\right.$, df $\left.=1, P=0.159\right)$. Two patients in DCEAS group discontinued due to intolerance of acupuncture stimulation.

\section{Credibility of sham and DCEAS procedure}

There was no significant difference in the credibility rating between the two groups, with $45.5 \%$ (15/33) of patients treated with n-EA perceiving to have received DCEAS, while $23.5 \%$ (8/ 34) of patients in DCEAS believed to have n-EA treatment $\left(\chi^{2}=2.665, P=0.103\right)$.

\section{Discussion}

The present study demonstrated that DCEAS intervention is effective in augmenting the antidepressant efficacy of FLX in the treatment of moderate and severe MDD. While the patients in the two groups had received similar FLX doses during the study, DCEAS-treated patients exhibited greater improvement on depressive symptoms, as indicated with the significant greater reduction of HAMD-17 and SDS score at most measure time points, although the magnitude of the reduction of SGI-S score, the response and remission rates were not different in the two groups. Moreover, the greater reduction of both HAMD-17 and SDS was observed as early as at day 3 after the first session of acupuncture treatment. Similar result was also present at endpoint of three weeks of DCEAS intervention. These data suggest that DCEAS intervention produces a rapid effect in alleviating depressive symptoms in both clinician-rated (HAMD-17) and self-rated (SDS) measures of depression. In addition, there were only two DCEAS-treated patients who discontinued due to intolerance of acupuncture. DCEAS intervention did not increase the incidence of any adverse events compared to n-EA control procedure, suggesting that DCEAS is a tolerable and safe stimulation mode.

While the current study showed the superior antidepressant efficacy of DCEAS over n-EA procedure when combined with FLX, several similar trials failed to demonstrate the superior effects of active acupuncture regimens in reducing depressive symptoms compared to sham and placebo acupuncture regimens [27-29]. This has raised the argument that the antidepressant benefits of acupuncture observed may be derived from placebo effects rather than physiological mechanisms [28]. Nevertheless, the present study revealed no significant difference in the credibility of the control and DCEAS procedures, with nearly $46 \%$ of n-EA-treated patients who perceived to have received active procedure, while $24 \%$ of DCEAS-treated patients perceived the control procedure, suggesting that the non-inserted needling stimulation used in the present study for a control procedure was valid and acceptable. In fact, previous studies have well demonstrated the high credibility of the non-inserted needle device [26]. Therefore, it was unlikely that the antidepressant benefits of DCEAS observed in the present study were derived from placebo effects.

There are two possible explanations for negative results in the previous acupuncture studies of major depression [27-29]. Firstly, unlike the present study that used the non-inserted control procedure, the previous studies needled at non-meridian-based acupoints which are located at a certain distances (usually $1-3 \mathrm{~cm}$ ) from the meridian-based acupoints [27-29]. Although there seems to be some differences in the histological profile between the meridian- and non-meridian-based acupoints [30], it might be difficult to differentiate the physiological responses induced by stimulation at the two types of acupoints. Second, relatively few acupoints were used in the previous studies [27-29]. This may result in inadequacy of acupuncture stimulation which is believed to be an important factor associated with negative results of acupuncture trials [31].

DCEAS was developed mainly based on a neurobiological rationale. It is well documented that the forehead acupoints innervated by the trigeminal sensory pathway have intimate collateral connections with the brainstem reticular formation, in particular the dorsal raphe nucleus (DRN) [32,33] and the locus coeruleus (LC) [34-37]. The latter two brain structures are the major resources of 5-HT and NA neuronal bodies, respectively, 

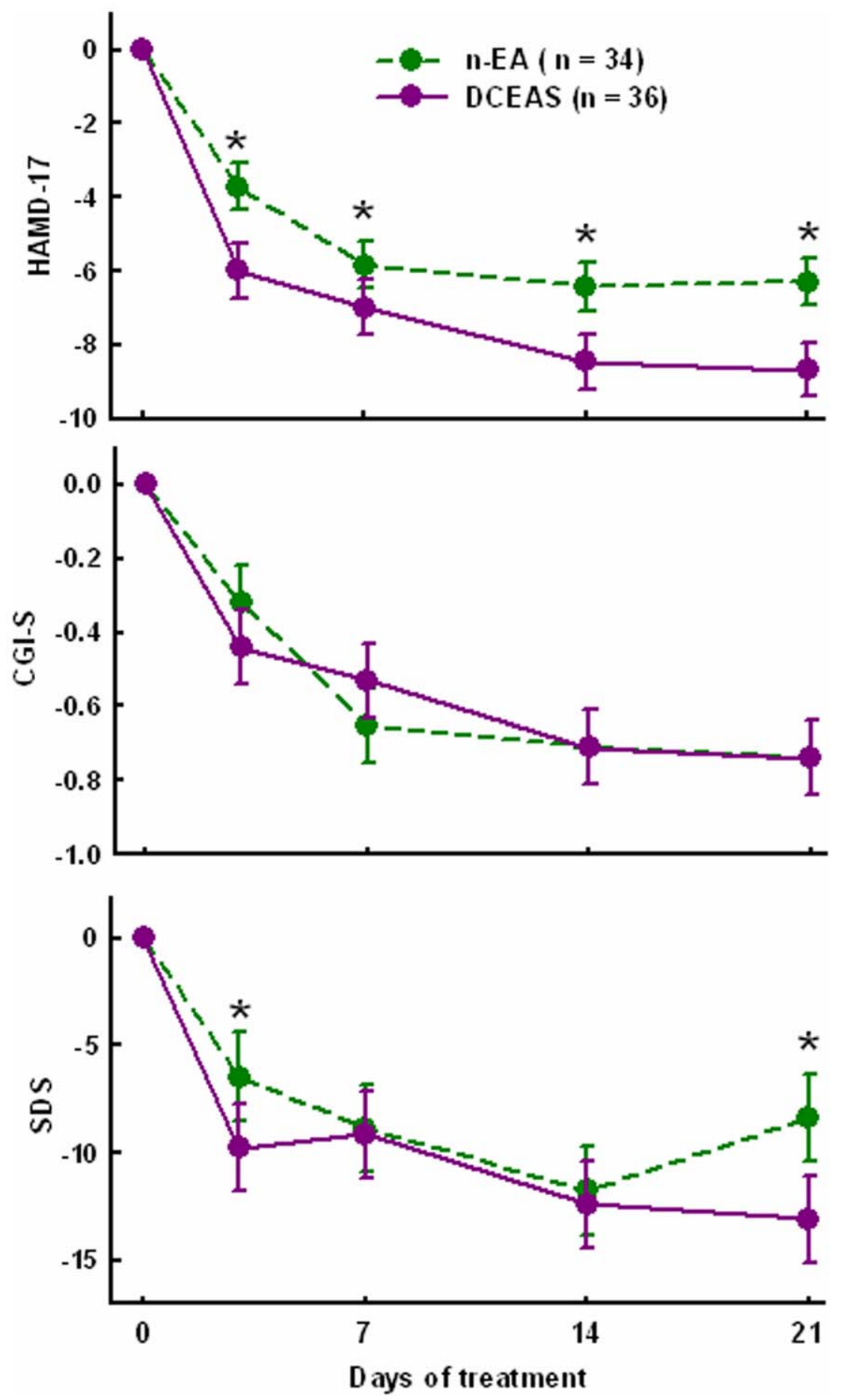

Figure 3. Mean changes from baseline in score on the 17-item Hamilton Rating Scale for Depression (HAMD-17), Clinical Global Impression-Severity (CGI-S) and Self-rating Depression Scale (SDS). Data are expressed as mean with $95 \%$ confidence interval (95\% CI). * $P \leq 0.037$ : between-group comparison using Student $t$-test. doi:10.1371/journal.pone.0029651.g003

sending diffuse projections to subcortical and cortical areas, including the prefrontal cortex and the amygdala known to be heavily involved in the pathogenesis of depressive disorders [38]. A large body of evidence confirms that the brainstem 5-HT and NA neuronal systems play a pivotal role in acupuncture modulation of multiple brain functions, including pain, emotion, sleep, and visceral information processing [8-10,39]. Neuroanatomical and neurophysiological studies have demonstrated that electroacupuncture stimulation increases the expression of 5-HT in the DRN [40] and suppresses the stress-induced increase in neuronal activities of the LC [41,42]. Neuroimaging studies have also shown that electroacupuncture stimulation is capable of directly modulating the activity of the emotion processing-related brain regions [8]. Through the intimate collateral connection from the trigeminal 
Table 3. Adverse events occurred in at least $5 \%$ of patients in either group.

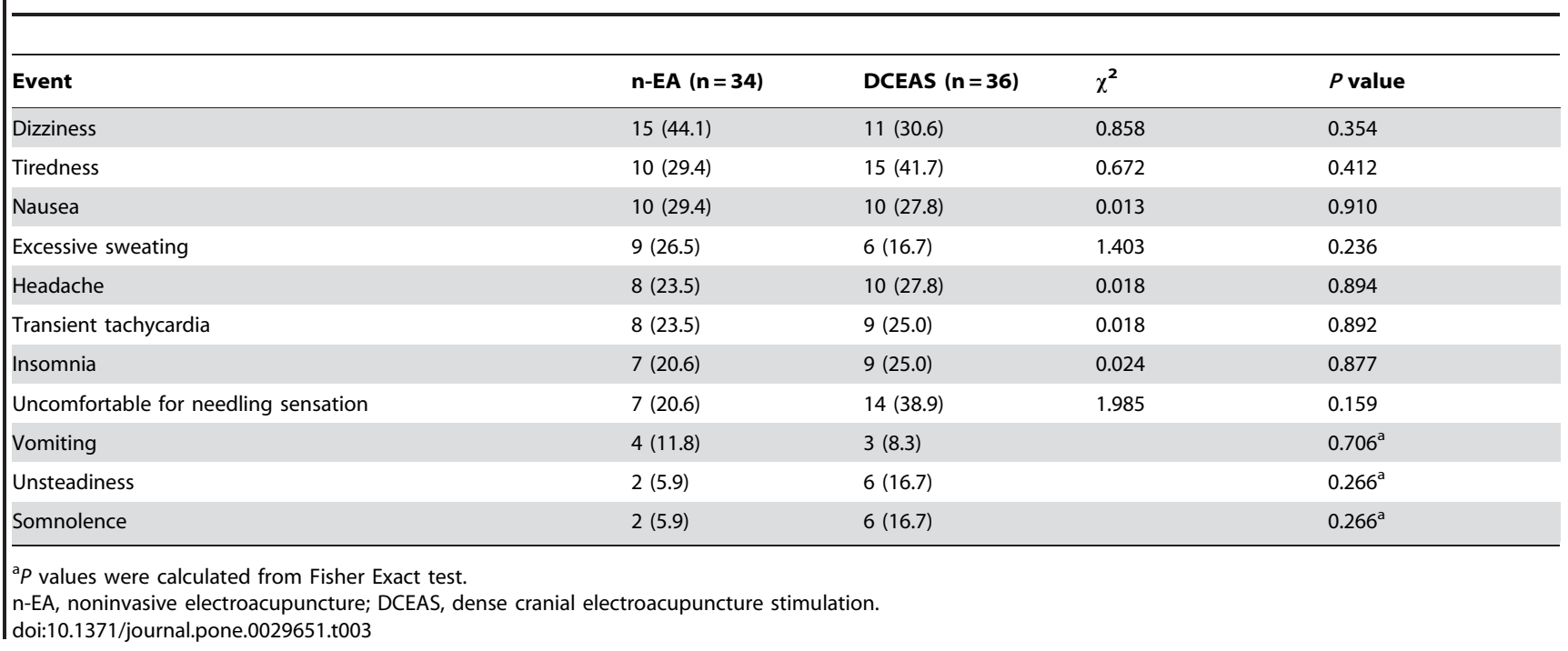

sensory pathway to the brainstem 5-HT and NA neuronal systems, the needling of the forehead acupoints with subsequent electrical stimulation could robustly elicit afferent acupuncture signals via biophysical and biochemical reactions at local acupoints and, in turn, efficiently modulates central 5-HT and NA neuronal functions $[9,10]$. On the other hand, like other noninvasive brain stimulation therapies, such as transcutaneous electrical nerve stimulation (TENS), repetitive transcranial magnetic stimulation (rTMS), and electroconvulsive therapy (ECT) [43,44], electrical stimulation was also directly delivered on the scalp in both control and DCEAS procedure. This may elicit a transcutaneous and/or transcranial effect; however, such effect would likely be minimal as the stimulation intensity used in both n-EA control and DCEAS procedures was generally much lower than rTMS, ECT, and most TENS $[43,44]$. Therefore, we have reason to believe that the antidepressant efficacy of DCEAS observed in the present study should be derived mainly from the biophysical and biochemical effects produced in needling with subsequent electrical stimulation [45]. This also could explain the superior therapeutic efficacy of DCEAS over n-EA control procedure.

Several limitations of the present study should be noticed. First, the study was conducted in a female-dominated sample with a significant difference in the proportion of female subjects between the two groups. Epidemiological evidence suggests that women are more likely to use complementary and alternative medicine (CAM) and have a higher degree of confidence in CAM efficacy and safety [46]. Whether similar therapeutic effects of DCEAS could be achieved in male patients needs to be further investigated. In addition, as the study was conducted in a single-blind manner, effects mediated by non-blinded acupuncturists could not be excluded. Recently, a well-demonstrated control method for single-blind condition has been introduced and could be considered in future studies [47]. Second, although DCEAS achieved a clinically meaningful, over 2 -fold difference in the response rate than the control procedure $(19.4 \%$ vs. $8.8 \%)$, the difference did not reach statistical significance level. This may be due to the relatively low response rates in the two groups when compared to other similar trials, with response rates of $22-80 \%$ for active acupuncture and $39-78 \%$ for sham or placebo-treated groups [27-29]. The relatively low response rate observed in the present study appears to be related to the short-term (3-week) treatment. As long-term effects of DCEAS were not evaluated in this study and a majority of depressed patients may be required for long-term treatment [48], long-term antidepressant efficacy of DCEAS may deserve to be further investigated. Finally, although we measured patients' platelet 5-HT contents at baseline and posttreatment (data not shown in this report), no significant changes in platelet 5-HT parameters were observed in DCEAS-treated patients compared to patients treated with n-EA, suggesting that acupuncture may have least effects on non-neuronal 5-HT systems. Evaluation of DCEAS effects in the brain 5-HT neuronal system may help gain new insight into central mechanisms responsible for DCEAs effects.

Collectively, the present study demonstrates that DCEAS is a safe and effective intervention in augmenting the antidepressant efficacy in the early phase of SSRI treatment. As patients with moderate and severe major depression have a higher risk of suicide and the worsening of symptoms in the early phase of SSRI treatment, DCEAS can be considered as an additional treatment option. The present study guarantees a larger-scale, multi-site trial to further determine the effectiveness of DCEAS as a viable and safe non-pharmacological augmentation for depressive disorders.

\section{Supporting Information}

\section{Ghecklist S1 GONSORT Checklist.} (PDF)

\section{Protocol S1 Trial Protocol.} (PDF)

\section{Acknowledgments}

We thank the following colleagues at Department of Psychiatry of Kowloon Hospital for their assistance in patient recruitment: Ka-Lik Kwan, Chun-Ting Chan, Man-Lui Chan, Chi-Kwan Cheung, Janice Chik, Lung-Kit Hui, Man-Man Kwan, Chee-Kin Lee, Kwok-Chuen Ng, Yin-Ting Ng, Ting-Keung Poon, Fu-Yin Tong, Wai-Ching Yan, KamHing Yeung, Tin-Yan Yeung, Mei-Kuen Frieda Shiu, Cheuk-Kin Tang, Pui-Shan Tse, Ngar-Fong Lam, See-Cheuk Fu, Chui-Lin Carol Ching, Ka-Fai Ho, Sau-Lai Tai, Sau-Ming Chan, Yiu-Kwun Law, and Yuk-Kwan Yvonne Kwong.

\section{Author Contributions}

Conceived and designed the experiments: Z-JZ RN Q-RT K-cY. Performed the experiments: SCM TYJL WW M-TW W-KAT. Analyzed the data: Z-JZ HKW. Wrote the paper: Z-JZ RN Q-RT K-FG EZ VTW. 


\section{References}

1. Arroll B, Macgillivray S, Ogston S, Reid I, Sullivan F, et al. (2005) Efficacy and tolerability of tricyclic antidepressants and SSRIs compared with placebo for treatment of depression in primary care: a meta-analysis. Ann Fam Med 3: 449-456.

2. Blier P, de Montigny C (1994) Current advances and trends in the treatment of depression. Trends Pharmacol Sci 15: 220-226.

3. Blier P (2003) The pharmacology of putative early-onset antidepressant strategies. Eur Neuropsychopharmacol 13: 57-66.

4. Adell A, Castro E, Celada P, Bortolozzi A, Pazos A, et al. (2005) Strategies for producing faster acting antidepressants. Drug Discov Today 10: 578-585.

5. Zhang ZJ, Chen HY, Yip KC, Ng R, Wong VT (2010) The effectiveness and safety of acupuncture therapy in depressive disorders: systematic review and meta-analysis. J Affect Disord 124: 9-21.

6. Smith CA, Hay PP, Macpherson H (2010) Acupuncture for depression. Cochrane Database Syst Rev 20: CD004046.

7. Wang H, Qi H, Wang BS, Cui YY, Zhu L, et al. (2008) Is acupuncture beneficial in depression: a meta-analysis of 8 randomized controlled trials? J Affect Disord 111: $125-134$.

8. Dhond RP, Kettner N, Napadow V (2007) Neuroimaging acupuncture effects in the human brain. Altern Complement Med 13: 603-616.

9. Zhao ZQ (2008) Neural mechanism underlying acupuncture analgesia. Prog Neurobiol 85: 355-375.

10. Ulett GA, Han S, Han JS (1998) Electroacupuncture: mechanisms and clinical application. Biol Psychiatry 44: 129-138.

11. Belmaker RH, Agam G (2008) Major depressive disorder. N Engl J Med 358: 55-68.

12. Zhang ZJ, Wang XY, Tan QR, Jin GX, Yao SM (2009) Electroacupuncture for refractory obsessive-compulsive disorder: a pilot waitlist-controlled trial. J Nerv Ment Dis 197: 619-22.

13. Huang Y, Gong W, Zou J, Zhao CH (2004) An SCL-90 analysis of scalp electroacupuncture treatment of major depressive episode. Shanghai Zhen Jiu Za Zhi 23: 5-7. (in Chinese with English abstract).

14. Li HJ, Zhong BL, Fan YP, Hu HT (2011) Acupuncture for post-stroke depression: a randomized controlled trial. Zhongguo Zhen Jiu 31: 3-6. (in Chinese with English abstract).

15. Yeung WF, Chung KF, Tso KC, Zhang SP, Zhang ZJ, et al. (2011) Electroacupuncture for residual insomnia associated with major depressive disorder: a randomized controlled trial. Sleep 34: 807-815.

16. Stark P, Hardison CD (1985) A review of multicenter controlled studies of fluoxetine vs. imipramine and placebo in outpatients with major depressive disorder. J Clin Psychiatry 46: 53-58.

17. American Psychiatric Association (1994) Diagnostic and statistical manual of mental disorders. 4th ed. Washington, DC: American Psychiatric Association Press.

18. Hamilton M (1960) A rating scale for depression. Journal of Neurology Neurosurgery and Psychiatry 23: 56-62.

19. Guy W (1976) ECDEU assessment manual for psychopharmacology. revised. Bethesda, MD: US Department of Health, Education, and Welfare.

20. Hong CJ, Hu WH, Chen CC, Hsiao GC, Tsai SJ, et al. (2003) : A double-blind, randomized, group-comparative study of the tolerability and efficacy of 6 weeks' treatment with mirtazapine or fluoxetine in depressed Chinese patients. J Clin Psychiat 64: 921-926.

21. Yu YW, Tsai SJ, Liou YJ, Hong CJ, Chen TJ (2006) Association study of two serotonin 1A receptor gene polymorphisms and fluoxetine treatment response in Chinese major depressive disorders. Eur Neuropsychopharmacol 16: 498-503.

22. Han JS (2003) Acupuncture: neuropeptide release produced by electrical stimulation of different frequencies. Trends Neurosci 26: 17-22.

23. Streitberger K, Kleinhenz J (1998) Introducing a placebo needle into acupuncture research. Lancet 352: 364-365.

24. Enck P, Klosterhalfen S, Zipfel S (2010) Acupuncture, psyche and the placebo response. Auton Neurosci 157: 68-73.

25. Lee HC, Chiu HF, Wing YK, Leung CM, Kwong PK, et al. (1994) The Zung Self-rating Depression Scale: screening for depression among the Hong Kong Chinese elderly. J Geriatr Psychiatry Neurol 7: 216-220.
26. Fink M, Gutenbrunner C, Rollnik J, Karst M (2001) Credibility of a newly designed placebo needle for clinical trials in acupuncture research. Forsch Komplementarmed Klass Naturheilkd 8: 368-372.

27. Allen JJ, Schnyer RN, Chambers AS, Hitt SK, Moreno FA, et al. (2006) Acupuncture for depression: a randomized controlled trial. J Clin Psychiatry 67: $1665-1673$.

28. Andreescu C, Glick RM, Emeremni CA, Houck PR, Mulsant BH (2011) Acupuncture for the treatment of major depressive disorder: a randomized controlled trial. J Clin Psychiatry 2011;72: 1129-1135.

29. Zhang WJ, Yang XB, Zhong BL (2009) Combination of acupuncture and fluoxetine for depression: a randomized, double-blind, sham-controlled trial. J Altern Complement Med 15: 837-844.

30. Zhou F, Huang D, Xia Y (2009) Neuroanatomical basis of acupuncture points (Chapter 2). In Xia Y, Wu G, Cao X, Chen J, eds. Acupuncture therapy for neurological diseases: a neurobiological view, Tsinghua University Press, Beijing, and Springer-Verlag Berlin, Heidelberg. pp 32-80.

31. Benham A, Johnson MI (2009) Could acupuncture needle sensation be a predictor of analgesic response? Acupunct Med 27: 65-67.

32. Arbab MA, Delgado T, Wiklund L, Svendgaard NA (1988) Brain stem terminations of the trigeminal and upper spinal ganglia innervation of the cerebrovascular system: WGA-HRP transganglionic study. J Cereb Blood Flow Metab 8: 54-63.

33. Kubota K, Narita N, Ohkubo K, Hosaka K, Nagae K, et al. (1988) Central projection of proprioceptive afferents arising from maxillo-facial regions in some animals studied by HRP-labeling technique. Anat Anz 165: 229-251.

34. Foote SL, Bloom FE, Aston-Jones G (1983) Nucleus locus ceruleus: new evidence of anatomical and physiological specificity. Physiol Rev 63: 844-914.

35. Simpson KL, Altman DW, Wang L, Kirifides ML, Lin RC, et al. (1997) Lateralization and functional organization of the locus coeruleus projection to the trigeminal somatosensory pathway in rat. J Comp Neurol 385: 135-147.

36. Simpson KL, Waterhouse BD, Lin RC (1999) Origin, distribution, and morphology of galaninergic fibers in the rodent trigeminal system. J Comp Neurol 411: 524-534.

37. Takahashi T, Shirasu M, Shirasu M, Kubo KY, Onozuka M, et al. (2010) The locus coeruleus projects to the mesencephalic trigeminal nucleus in rats. Neurosci Res 68: 103-106.

38. Clark L, Chamberlain SR, Sahakian BJ (2009) Neurocognitive mechanisms in depression: implications for treatment. Annu Rev Neurosci 32: 57-74.

39. Takahashi $\mathrm{T}$ (2006) Acupuncture for functional gastrointestinal disorders. J Gastroenterol 41: 408-417.

40. Kwon YB, Kang MS, Son SS, Kim JT, Lee YH, et al. (2000) Different frequencies of electroacupuncture modified the cellular activity of serotonergic neurons in brainstem. Am J Chin Med 28: 435-441.

41. Lee HJ, Lee B, Choi SH, Hahm DH, Kim MR, et al. (2004) Electroacupuncture reduces stress-induced expression of c-fos in the brain of the rat. Am J Chin Med 32: 795-806.

42. Li A, Wang Y, Xin J, Lao L, Ren K, et al. (2007) Electroacupuncture suppresses hyperalgesia and spinal Fos expression by activating the descending inhibitory system. Brain Res 1186: 171-179.

43. Kirkcaldie M, Pridmore S, Reid P (1997) Bridging the skull: electroconvulsive therapy (ECT) and repetitive transcranial magnetic stimulation (rTMS) in psychiatry. Convulsive Therapy 13: 83-91.

44. van Dijk KR, Scherder EJ, Scheltens P, Sergeant JA (2002) Effects of transcutaneous electrical nerve stimulation (TENS) on non-pain related cognitive and behavioural functioning. Rev Neurosci 13: 257-270.

45. Zhang ZJ, Wang XM, McAlonan GM (2011) Neural acupuncture unit (NAU): a new concept for interpreting effects and mechanisms of acupuncture. eCAM (accepted, Article ID: 429412).

46. Barnes PM, Powell-Griner E, McFann K, Nahin RL (2004) Complementary and alternative medicine use among adults: United States, Adv Data 27: 1-19.

47. La Marca R, Nedeljkovic M, Yuan L, Maercker A, Ehlert U (2010) Effects of auricular electrical stimulation on vagal activity in healthy men: Evidence from a three-armed randomized trial. Clin Sci (Lond) 118: 537-546.

48. Keller MB, Berndt ER (2002) Depression treatment: a lifelong commitment? Psychopharmacol Bull 36(S2): 133-141. 